\title{
Comparative Microstructural Analysis of Non-graphitic Carbons by Wide-angle X-ray and Neutron Scattering
}

Torben Pfaff ${ }^{* 1}$, Felix M. Badaczewskii ${ }^{*}$, Marc O. Loeh ${ }^{1,2}$, Alexandra Franz ${ }^{3}$, Jens-Uwe Hoffmann ${ }^{3}$, Manfred Reehuis ${ }^{3}$, Wolfgang G. Zeier ${ }^{1}$, and Bernd M. Smarsly ${ }^{1,4}$

${ }^{1}$ Institute of Physical Chemistry, Justus Liebig University, Heinrich-Buff-Ring 17, D-35392 Giessen, Germany

${ }^{2}$ Schunk Carbon Technology, Schunk GmbH, D-35452 Heuchelheim, Germany ${ }^{3}$ Helmholtz Zentrum Berlin für Materialien und Energie, D-14109 Berlin, Germany ${ }^{4}$ Center for Materials Research (LaMa), Justus Liebig University, Heinrich-Buff-Ring 16, D-35392 Giessen, Germany 


\section{Parameters used by Ruland's \& Smarsly's algorithm for fitting NGC WAXS/WANS data}

Fig S1 explains the parameters used by Ruland's \& Smarsly's algorithm for fitting NGC WAXS/WANS data. The parameters are divided in parameters describing the layer structure, parameters describing the stacking and parameters describing impurities.

\begin{tabular}{|c|c|c|c|}
\hline & Parameter & Denotation & Explanation \\
\hline \multirow{5}{*}{ 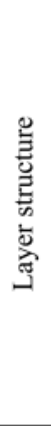 } & $L_{a}{ }^{b}$ & Average graphene layer extent & \\
\hline & $\langle l\rangle^{b}$ & Average chord length & \\
\hline & $\kappa_{a}^{b}$ & Polydispersity of chord length $l$ & \\
\hline & $l_{c c}$ & Average $\mathrm{C}-\mathrm{C}$ bond length & \\
\hline & $\sigma_{1}$ & $\begin{array}{l}\text { Standard deviation of the first- } \\
\text { neighbor distribution }\end{array}$ & \\
\hline \multirow{10}{*}{ 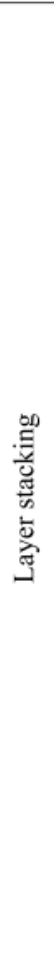 } & $L_{c}{ }^{c}$ & Average stack height & \multirow{2}{*}{ 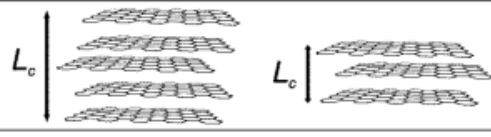 } \\
\hline & $\kappa_{c}{ }^{c}$ & Polydispersity of stack height & \\
\hline & $a_{3}$ & Average interlayer spacing & \multirow{4}{*}{ 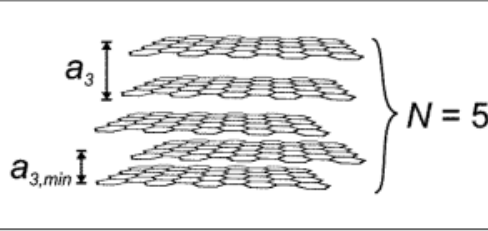 } \\
\hline & $a_{3, \min }$ & Minimum of interlayer spacing & \\
\hline & $\sigma_{3}$ & $\begin{array}{l}\text { Standard deviation of interlayer } \\
\text { spacing }\end{array}$ & \\
\hline & $\langle N\rangle^{c}$ & Number of graphene layers per stack & \\
\hline & $u_{3}$ & Parameter of thermal motion & \\
\hline & $\eta$ & Homogeneity of the stacks & 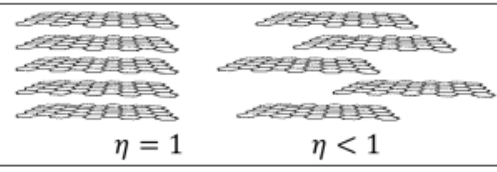 \\
\hline & $q$ & Parameter of preferred orientation & 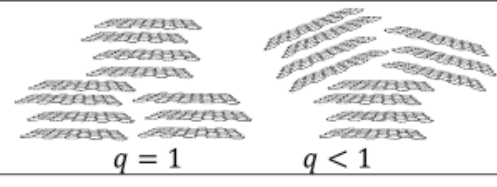 \\
\hline & $\Delta_{a n}$ & $\begin{array}{l}\text { Parameter of anisotropy of the } \\
\text { atomic form factor of carbon }\end{array}$ & $\underset{2 \sin (\theta) / \lambda}{\stackrel{f_{C}}{\mathrm{f}_{\mathrm{C}}}} \underset{\substack{\text { to graphene } \\
\text { layer }}}{\text { Atomic form factor }}$ \\
\hline \multirow{3}{*}{ : } & $c_{u n}$ & $\begin{array}{l}\text { Concentration of unorganized } \\
\text { carbon }\end{array}$ & \\
\hline & $c_{N}$ & $\begin{array}{l}\text { Concentration of unorganized } \\
\text { nitrogen }\end{array}$ & \\
\hline & $c_{O}$ & $\begin{array}{l}\text { Concentration of unorganized } \\
\text { oxygen }\end{array}$ & \\
\hline
\end{tabular}

Figure S1: Overview of the 14 microstructural parameters of non-graphitic-carbons obtainable by fitting the WAXS data according to the model of Ruland and Smarsly (Ruland and Smarsly, 2002) and further parameters considered for correct modelling. The represented parameters are not mathematically independent. Reprinted with permission from J. Phys. Chem. C 2014, 118, 29, 15705-15715. Copyright(2014) American Chemical Society.

b These parameters are dependent, since the shape factors $\nu$ and $\alpha$, which describe a gamma distribution, are used for their calculation. ${ }^{\mathrm{c}}$ These parameters are dependent, since the shape factors $\mu$ and $\beta$, which describe a gamma distribution, are used for their calculation. 


\section{Overview of the acquired wide-angle scattering curves}

The three figures below depict an overview of all acquired wide-angle scattering curves. In Fig S2 all WAXS curves acquired by using an standard laboratory XRD setup are shown. Fig S3 contains all WANS curves. Please note that two different WANS instruments, the E2 and E9 instrument of Helmholtz-Zentrum Berlin, Germany, having a different range of $s=\frac{2 \cdot \sin \theta}{\lambda}$ were used. Fig S4 displays all WAXS curves for PDF analysis acquired at the XPDF beamline of Diamond, UK.

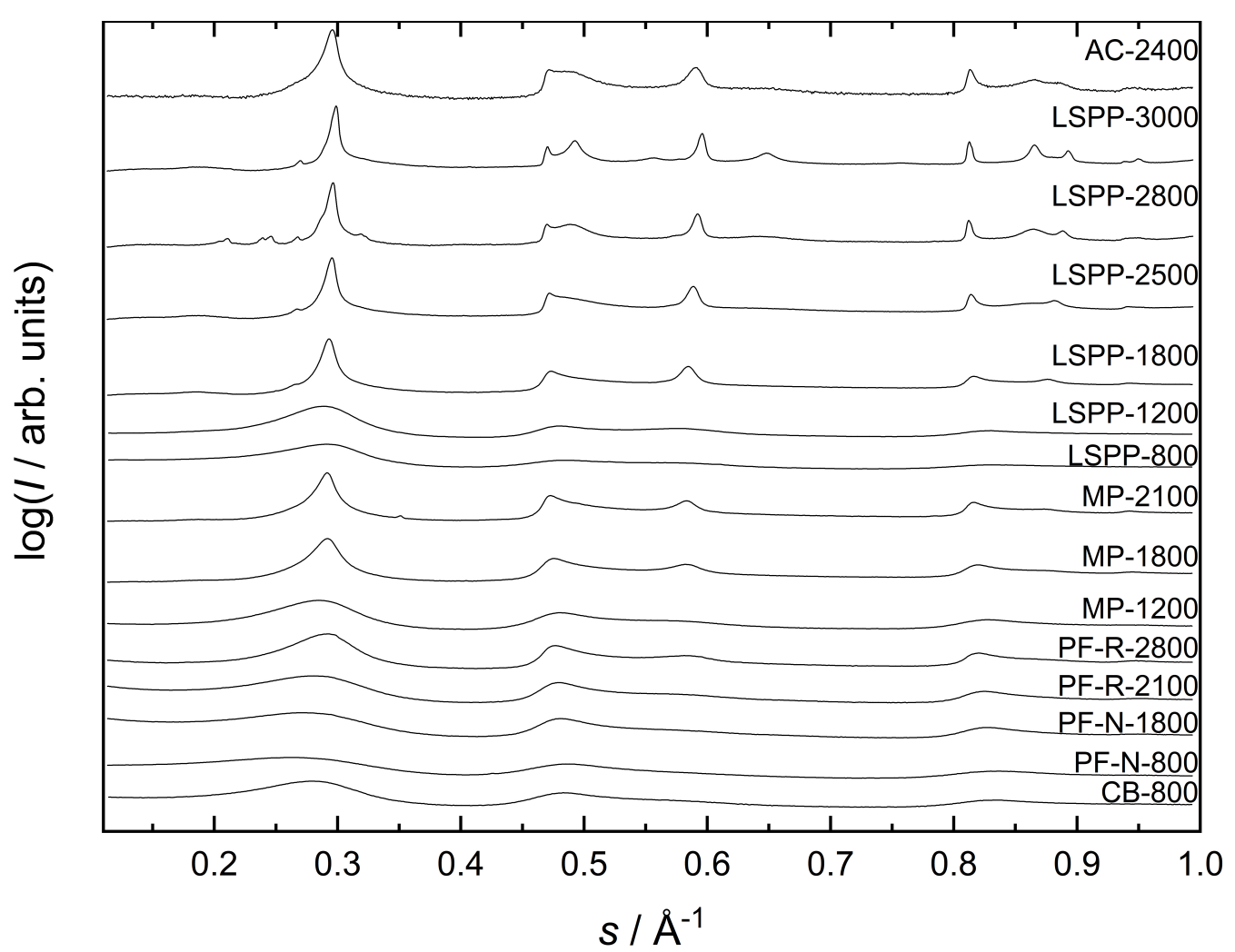

Figure S2: Overview of all acquired WAXS curves by using an standard laboratory XRD setup. 


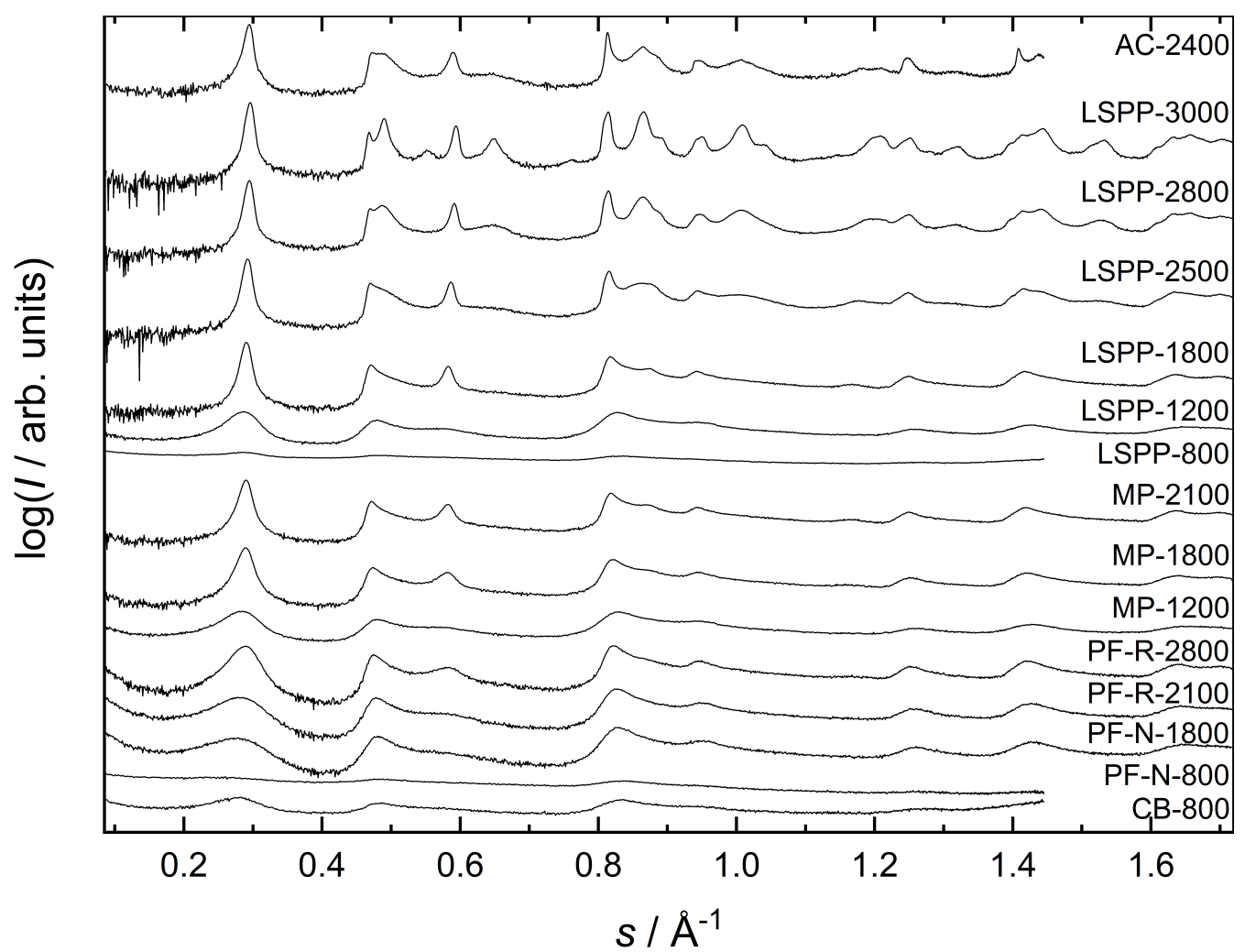

Figure S3: Overview of all acquired WANS curves by using an the E2 and E9 instrument of HelmholtzZentrum Berlin, Germany.

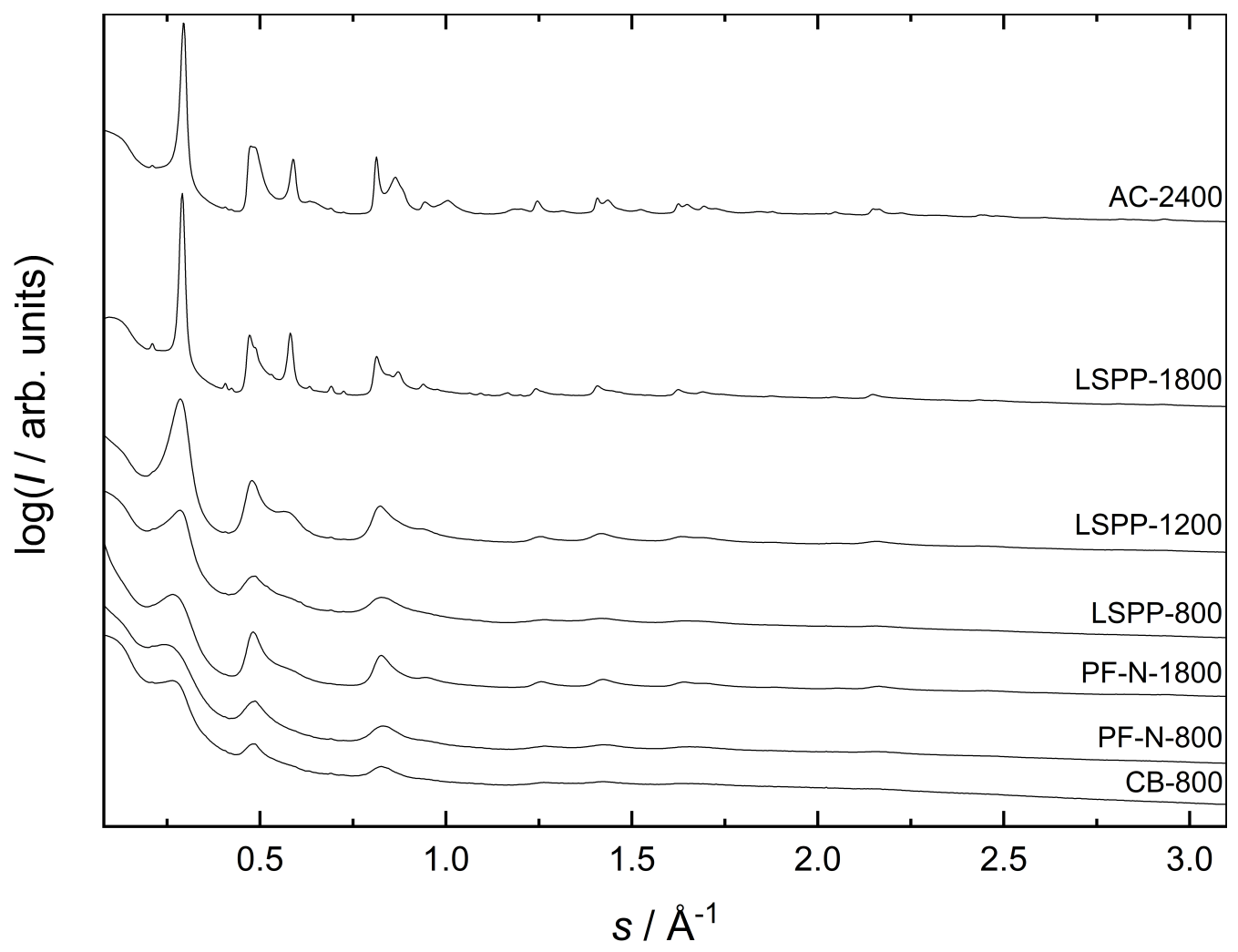

Figure S4: Overview of all acquired WAXS curves for PDF analysis by using the XPDF beamline of Diamond, UK. 


\section{WAXS/WANS Comparison}

In this section, the parameters obtained from fitting the WANS and WAXS data are given in Table S1.

For a detailed discussion of these results please refer to the main article.

Table S1: Overview of the parameters obtained by automatic fitting in CarbX. All values are given in $\AA$ except for $\sigma_{1}$, being dimensionless.

\begin{tabular}{llll} 
& \multicolumn{1}{c}{ LSPP-800 } & \multicolumn{1}{c}{ PF-N-800 } & \multicolumn{1}{c}{ CB-800 } \\
& \multicolumn{1}{c}{ WAXS } & \multicolumn{1}{c}{ WAXS } & \multicolumn{1}{c}{ WAXS } \\
$L_{\mathrm{a}}$ & $17 \pm 2.6$ & $20 \pm 3.0$ & $26 \pm 3.9$ \\
$l_{\mathrm{cc}}$ & $1.409 \pm 0.006$ & $1.407 \pm 0.006$ & $1.409 \pm 0.006$ \\
$\sigma_{1}$ & $0.15 \pm 0.018$ & $0.14 \pm 0.016$ & $0.14 \pm 0.017$ \\
$L_{\mathrm{c}}$ & $12 \pm 1.8$ & $7 \pm 1.1$ & $14 \pm 2.1$ \\
$\bar{a}_{3}$ & $3.42 \pm 0.05$ & $3.57 \pm 0.05$ & $3.57 \pm 0.05$ \\
$\sigma_{3}$ & $0.24 \pm 0.024$ & $0.43 \pm 0.043$ & $0.44 \pm 0.044$
\end{tabular}

\begin{tabular}{lllll}
\hline & \multicolumn{1}{c}{ LSPP-1200 } & \multicolumn{1}{c}{ LSPP-1200 } & \multicolumn{1}{c}{ MP-1200 } & \multicolumn{1}{c}{ MP-1200 } \\
& \multicolumn{1}{c}{ WAXS } & \multicolumn{1}{c}{ WANS } & \multicolumn{1}{c}{ WAXS } & \multicolumn{1}{c}{ WANS } \\
\hline$L_{\mathrm{a}}$ & $39 \pm 6$ & $27 \pm 4.0$ & $34 \pm 5.1$ & $23 \pm 3.5$ \\
$l_{\mathrm{cc}}$ & $1.412 \pm 0.006$ & $1.418 \pm 0.006$ & $1.413 \pm 0.006$ & $1.417 \pm 0.006$ \\
$\sigma_{1}$ & $0.13 \pm 0.02$ & $0.062 \pm 0.007$ & $0.12 \pm 0.014$ & $0.059 \pm 0.007$ \\
$L_{\mathrm{c}}$ & $19 \pm 3$ & $17 \pm 2.5$ & $15 \pm 2.3$ & $18 \pm 2.7$ \\
$\bar{a}_{3}$ & $3.45 \pm 0.05$ & $3.48 \pm 0.05$ & $3.49 \pm 0.05$ & $3.53 \pm 0.05$ \\
$\sigma_{3}$ & $0.22 \pm 0.02$ & $0.31 \pm 0.03$ & $0.27 \pm 0.027$ & $0.42 \pm 0.042$
\end{tabular}

\begin{tabular}{lllllll}
\hline & \multicolumn{1}{c}{ LSPP-1800 } & \multicolumn{1}{c}{ LSPP-1800 } & \multicolumn{1}{c}{ MP-1800 } & \multicolumn{1}{c}{ MP-1800 } & \multicolumn{1}{c}{ PF-N-1800 } & \multicolumn{1}{c}{ PF-N-1800 } \\
& \multicolumn{1}{c}{ WAXS } & \multicolumn{1}{c}{ WANS } & \multicolumn{1}{c}{ WAXS } & \multicolumn{1}{c}{ WANS } & \multicolumn{1}{c}{ WAXS } & \multicolumn{1}{c}{ WANS } \\
\hline$L_{\mathrm{a}}$ & $163 \pm 20$ & $126 \pm 20$ & $42 \pm 6.3$ & $48 \pm 7.2$ & $42 \pm 6$ & $32 \pm 5$ \\
$l_{\mathrm{cc}}$ & $1.417 \pm 0.006$ & $1.421 \pm 0.006$ & $1.412 \pm 0.006$ & $1.420 \pm 0.006$ & $1.412 \pm 0.006$ & $1.413 \pm 0.006$ \\
$\sigma_{1}$ & $0.061 \pm 0.007$ & $0.032 \pm 0.004$ & $0.10 \pm 0.012$ & $0.050 \pm 0.006$ & $0.061 \pm 0.007$ & $0.065 \pm 0.008$ \\
$L_{\mathrm{c}}$ & $150 \pm 20$ & $100 \pm 20$ & $70 \pm 11$ & $44 \pm 6.6$ & $9.2 \pm 1$ & $13 \pm 2$ \\
$\bar{a}_{3}$ & $3.42 \pm 0.05$ & $3.43 \pm 0.05$ & $3.44 \pm 0.05$ & $3.47 \pm 0.005$ & $3.54 \pm 0.05$ & $3.65 \pm 0.05$ \\
$\sigma_{3}$ & $0.11 \pm 0.01$ & $0.12 \pm 0.1$ & $0.28 \pm 0.028$ & $0.22 \pm 0.022$ & $0.28 \pm 0.03$ & $0.57 \pm 0.057$
\end{tabular}

\begin{tabular}{lllll}
\hline & \multicolumn{1}{c}{ MP-2100 } & \multicolumn{1}{c}{ MP-2100 } & \multicolumn{1}{c}{ PF-R-2100 } & \multicolumn{1}{c}{ PF-R-2100 } \\
& \multicolumn{1}{c}{ WAXS } & \multicolumn{1}{c}{ WANS } & \multicolumn{1}{c}{ WAXS } & \multicolumn{1}{c}{ WANS } \\
\hline$L_{\mathrm{a}}$ & $130 \pm 20$ & $77 \pm 10$ & $51 \pm 8$ & $40 \pm 6$ \\
$l_{\mathrm{cc}}$ & $1.417 \pm 0.006$ & $1.417 \pm 0.006$ & $1.413 \pm 0.006$ & $1.413 \pm 0.006$ \\
$\sigma_{1}$ & $0.066 \pm 0.008$ & $0.036 \pm 0.004$ & $0.097 \pm 0.01$ & $0.067 \pm 0.008$ \\
$L_{\mathrm{c}}$ & $84 \pm 10$ & $81 \pm 10$ & $11 \pm 2$ & $14 \pm 2$ \\
$\bar{a}_{3}$ & $3.44 \pm 0.05$ & $3.45 \pm 0.05$ & $3.49 \pm 0.05$ & $3.56 \pm 0.05$ \\
$\sigma_{3}$ & $0.13 \pm 0.01$ & $0.14 \pm 0.01$ & $0.22 \pm 0.02$ & $0.44 \pm 0.04$
\end{tabular}

\begin{tabular}{lll}
\hline & \multicolumn{1}{c}{$\begin{array}{c}\text { PF-R-2800 } \\
\text { WAXS }\end{array}$} & \multicolumn{1}{c}{$\begin{array}{c}\text { PF-R-2800 } \\
\text { WANS }\end{array}$} \\
\hline$L_{\mathrm{a}}$ & $87 \pm 13$ & $51 \pm 7.7$ \\
$l_{\mathrm{cc}}$ & $1.414 \pm 0.006$ & $1.419 \pm 0.006$ \\
$\sigma_{1}$ & $0.081 \pm 0.010$ & $0.047 \pm 0.006$ \\
$L_{\mathrm{c}}$ & $19 \pm 2.9$ & $23 \pm 3.5$ \\
$\bar{a}_{3}$ & $3.44 \pm 0.05$ & $3.48 \pm 0.05$ \\
$\sigma_{3}$ & $0.16 \pm 0.016$ & $0.25 \pm 0.025$
\end{tabular}




\section{Scherrer analysis}

As the samples exposed to higher temperatures $\left(2500,2800\right.$ and $3000{ }^{\circ} \mathrm{C}$ ) exhibit reflections of graphitic carbon, which prevent the application of of the analytical approach of Ruland/Smarsly, the (001) reflection of these samples WAXS and WANS data were analysed using the Scherrer equation. The results of these analyses are given below in Table S2 and S5. For a detailed discussion of these results please refer to the main article.

Table S2: Overview of the parameters obtained by applying the Scherrer equation. All values are given in $\AA$.

\begin{tabular}{ccccc}
\hline & $\begin{array}{c}\text { AC-2400 } \\
\text { WAXS }\end{array}$ & $\begin{array}{c}\text { AC-2400 } \\
\text { WANS }\end{array}$ & $\begin{array}{c}\text { LSPP-2500 } \\
\text { WAXS }\end{array}$ & $\begin{array}{c}\text { LSPP-2500 } \\
\text { WANS }\end{array}$ \\
\hline$L_{\mathrm{c}}$ & 84 & 72 & 130 & 72 \\
& & & & \\
\hline & LSPP-2800 & LSPP-2800 & LSPP-3000 \\
WAXS & WANS & WAXS & WANS \\
\hline$L_{\mathrm{c}}$ & 149 & 77 & 171 & 78
\end{tabular}

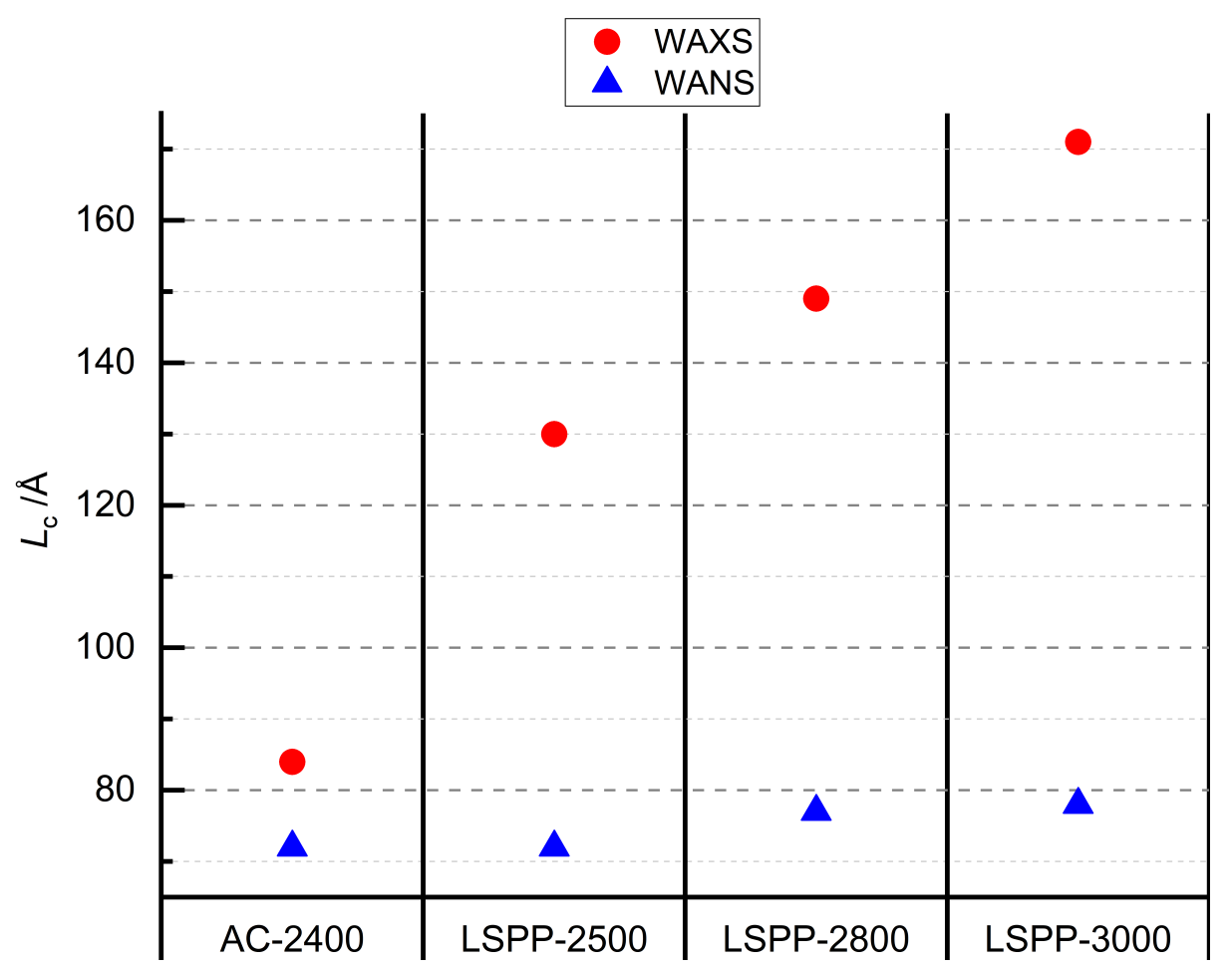

Figure S5: Comparison of $L_{\mathrm{c}}$ for four samples made from graphitizing low softening point pitch (LSPP2500, LSPP-2800, LSPP-3000) and acetylene coke (AC-2400). 


\section{$5 \quad$ Simulated Data}

In this section, the parameters yielded from fitting the impaired simulated data are given in Tables S3 and S4 along with the parameters used for simulation. For a detailed discussion of these results please refer to the main article.

Table S3: Overview of the parameters used for data simulation and those obtained by automatic fitting the quality-impaired WANS data. All values are given in $\AA$ except for $\sigma_{1}$, being dimensionless.

\begin{tabular}{|c|c|c|c|c|c|c|c|c|c|c|c|c|c|}
\hline & noise-level & \multicolumn{6}{|c|}{0.00} & \multicolumn{6}{|c|}{0.017} \\
\hline & cut-off / $\AA^{-1}$ & 0.8 & 1.2 & 1.8 & 2.4 & 3.0 & 3.6 & 0.8 & 1.2 & 1.8 & 2.4 & 3.0 & 3.6 \\
\hline parameter & simulation value & & & & & & & & & & & & \\
\hline$L_{\mathrm{a}}$ & 29 & 29 & 29 & 29 & 29 & 29 & 29 & 28 & 29 & 29 & 28 & 28 & 28 \\
\hline$l_{\mathrm{cc}}$ & 1.417 & 1.417 & 1.417 & 1.417 & 1.417 & 1.417 & 1.417 & 1.417 & 1.417 & 1.417 & 1.417 & 1.417 & 1.417 \\
\hline$\sigma_{1}$ & 0.075 & 0.075 & 0.075 & 0.075 & 0.075 & 0.075 & 0.075 & 0.078 & 0.079 & 0.079 & 0.078 & 0.077 & 0.077 \\
\hline$L_{\mathrm{c}}$ & 39 & 39 & 39 & 39 & 39 & 39 & 39 & 40 & 40 & 40 & 40 & 40 & 40 \\
\hline $\bar{a}_{3}$ & 3.49 & 3.49 & 3.49 & 3.49 & 3.49 & 3.49 & 3.49 & 3.50 & 3.50 & 3.50 & 3.50 & 3.50 & 3.50 \\
\hline$\sigma_{3}$ & 0.28 & 0.28 & 0.28 & 0.28 & 0.28 & 0.28 & 0.28 & 0.29 & 0.29 & 0.29 & 0.29 & 0.29 & 0.29 \\
\hline & noise-level & & & 0.0 & & & & & & 0. & & & \\
\hline & cut-off / $\AA^{-1}$ & 0.8 & 1.2 & 1.8 & 2.4 & 3.0 & 3.6 & 0.8 & 1.2 & 1.8 & 2.4 & 3.0 & 3.6 \\
\hline parameter & simulation value & & & & & & & & & & & & \\
\hline$L_{\mathrm{a}}$ & 29 & 28 & 28 & 28 & 27 & 27 & 27 & 26 & 27 & 27 & 26 & 25 & 26 \\
\hline$l_{\mathrm{cc}}$ & 1.417 & 1.417 & 1.417 & 1.417 & 1.416 & 1.416 & 1.416 & 1.416 & 1.416 & 1.416 & 1.416 & 1.415 & 1.416 \\
\hline$\sigma_{1}$ & 0.075 & 0.082 & 0.082 & 0.085 & 0.080 & 0.079 & 0.079 & 0.086 & 0.085 & 0.090 & 0.083 & 0.080 & 0.081 \\
\hline$L_{\mathrm{c}}$ & 39 & 41 & 41 & 41 & 41 & 41 & 41 & 42 & 42 & 43 & 42 & 42 & 42 \\
\hline $\bar{a}_{3}$ & 3.49 & 3.50 & 3.50 & 3.50 & 3.50 & 3.50 & 3.50 & 3.51 & 3.51 & 3.51 & 3.51 & 3.51 & 3.51 \\
\hline$\sigma_{3}$ & 0.28 & 0.31 & 0.30 & 0.31 & 0.30 & 0.30 & 0.30 & 0.32 & 0.31 & 0.32 & 0.32 & 0.31 & 0.31 \\
\hline
\end{tabular}


Table S4: Overview of the parameters used for data simulation and those obtained by automatic fitting the quality-impaired WAXS data. All values are given in $\AA$ except for $\sigma_{1}$, being dimensionless.

\begin{tabular}{|c|c|c|c|c|c|c|c|c|c|c|c|c|c|}
\hline & noise-level & \multicolumn{6}{|c|}{0.00} & \multicolumn{6}{|c|}{0.017} \\
\hline & cut-off / $\AA^{-1}$ & 0.8 & 1.2 & 1.8 & 2.4 & 3.0 & 3.6 & 0.8 & 1.2 & 1.8 & 2.4 & 3.0 & 3.6 \\
\hline parameter & simulation value & & & & & & & & & & & & \\
\hline$L_{\mathrm{a}}$ & 29 & 29 & 29 & 29 & 29 & 29 & 29 & 27 & 27 & 27 & 27 & 27 & 27 \\
\hline$l_{\mathrm{cc}}$ & 1.417 & 1.417 & 1.417 & 1.417 & 1.417 & 1.417 & 1.417 & 1.417 & 1.417 & 1.417 & 1.417 & 1.416 & 1.417 \\
\hline$\sigma_{1}$ & 0.075 & 0.075 & 0.075 & 0.075 & 0.075 & 0.075 & 0.075 & 0.073 & 0.073 & 0.077 & 0.077 & 0.078 & 0.074 \\
\hline$L_{\mathrm{c}}$ & 39 & 39 & 39 & 39 & 39 & 39 & 39 & 41 & 41 & 41 & 41 & 41 & 41 \\
\hline $\bar{a}_{3}$ & 3.49 & 3.49 & 3.49 & 3.49 & 3.49 & 3.49 & 3.49 & 3.50 & 3.49 & 3.49 & 3.49 & 3.49 & 3.49 \\
\hline$\sigma_{3}$ & 0.28 & 0.28 & 0.28 & 0.28 & 0.28 & 0.28 & 0.28 & 0.30 & 0.29 & 0.29 & 0.29 & 0.29 & 0.29 \\
\hline
\end{tabular}

\begin{tabular}{|c|c|c|c|c|c|c|c|c|c|c|c|c|c|}
\hline & noise-level & \multicolumn{6}{|c|}{0.033} & \multicolumn{6}{|c|}{0.05} \\
\hline & cut-off / $\AA^{-1}$ & 0.8 & 1.2 & 1.8 & 2.4 & 3.0 & 3.6 & 0.8 & 1.2 & 1.8 & 2.4 & 3.0 & 3.6 \\
\hline parameter & simulation value & & & & & & & & & & & & \\
\hline$L_{\mathrm{a}}$ & 29 & 24 & 25 & 25 & 25 & 25 & 25 & 22 & 22 & 23 & 23 & 22 & 23 \\
\hline$l_{\mathrm{cc}}$ & 1.417 & 1.415 & 1.417 & 1.416 & 1.416 & 1.416 & 1.416 & 1.415 & 1.416 & 1.415 & 1.415 & 1.414 & 1.415 \\
\hline$\sigma_{1}$ & 0.075 & 0.079 & 0.071 & 0.078 & 0.078 & 0.080 & 0.070 & 0.072 & 0.068 & 0.079 & 0.078 & 0.080 & 0.066 \\
\hline$L_{\mathrm{c}}$ & 39 & 42 & 42 & 42 & 42 & 42 & 42 & 46 & 42 & 43 & 43 & 43 & 42 \\
\hline $\bar{a}_{3}$ & 3.49 & 3.50 & 3.50 & 3.50 & 3.50 & 3.50 & 3.50 & 3.51 & 3.50 & 3.50 & 3.50 & 3.50 & 3.50 \\
\hline$\sigma_{3}$ & 0.28 & 0.31 & 0.30 & 0.30 & 0.30 & 0.30 & 0.29 & 0.32 & 0.31 & 0.30 & 0.30 & 0.30 & 0.30 \\
\hline
\end{tabular}




\section{PDF simulation}

In this section, the results of fitting the PDF data are presented. Fig S6 shows the comparison of a simulated PDF of a graphite with the relatively ordered AC-2400 sample and the more disordered PF-N-2800 sample. Table S5 and Figures S7-S9 contain the result of the test on how fixing a parameter influences the fit quality and parameters obtained from it. In Table S6 the results of fitting the PDF up to 20 and $80 \AA$ are given. For a detailed discussion of these results please refer to the main article.

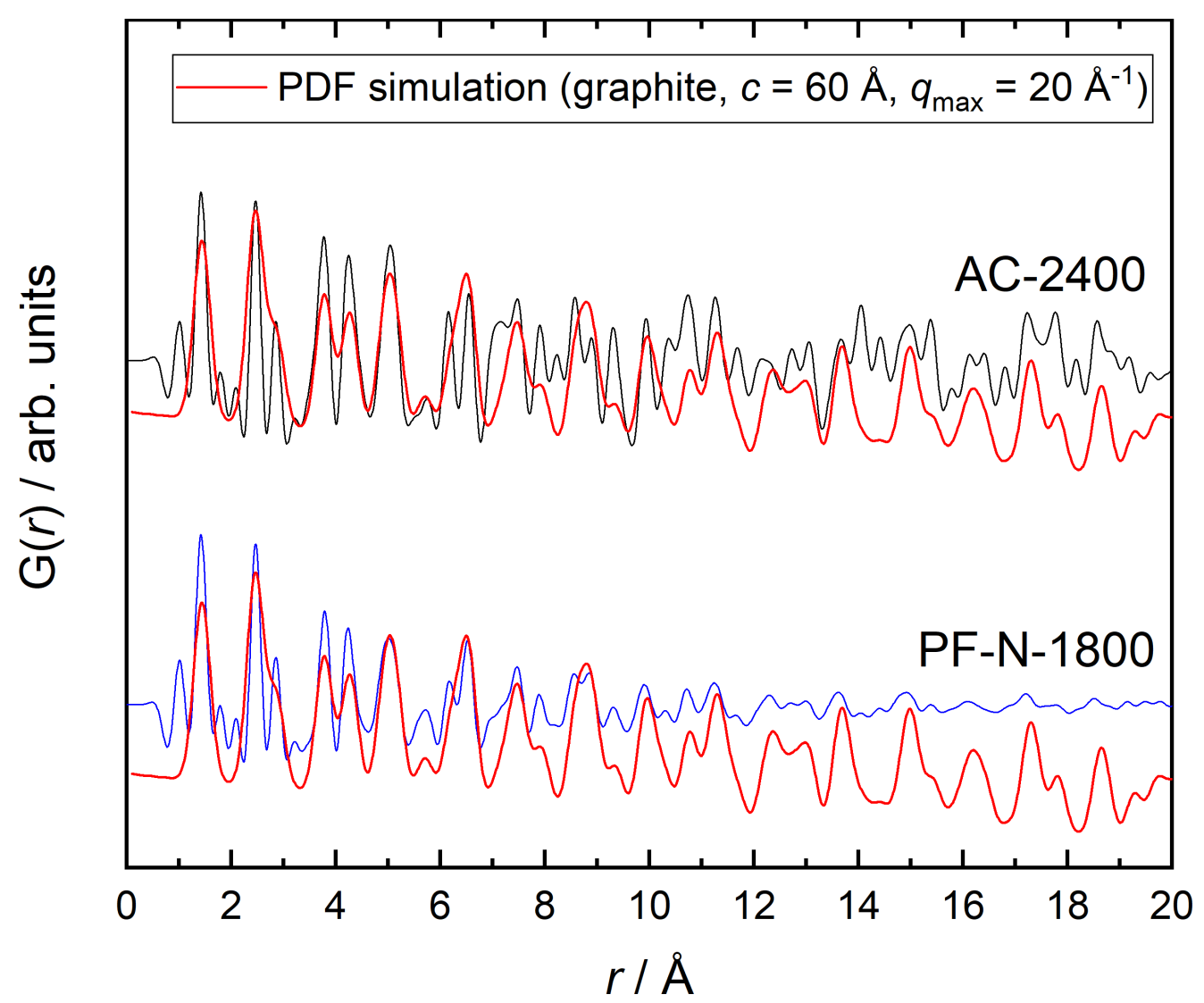

Figure S6: Comparison of a simulated PDF (red curve) utilizing the cif file of graphite having the layer distance enhanced to $60 \AA$ and the same cut-off as for the experimental data $\left(q_{\max }=20 \AA^{-1}\right)$ with two samples, the relatively ordered AC-2400 (black curve) and the more disordered PF-N-1800 (blue curve). By comparing the PDF of the respective sample with the simulated curve ripples can be identified. All maxima of the black/blue curve at positions where the red curve does not exhibit a maximum are ripples. The most pronounced ripples can be found at distances between one $(\sim 1.4 \AA)$ and two $(\sim 2.8$ A) C-C bond lengths in NGCs.

Table S5: Overview of the parameters obtained by fitting the PDF data of the PF-N-1800 sample up to $20 \AA$ as described in ${ }^{1}$.

\begin{tabular}{c|c|c|c}
$c / \AA$ & $u_{33} / \AA^{2}$ & $R_{\mathrm{w}} / \%$ & $s p$ diameter $/ \AA$ \\
\hline 8.0 & 1.4 & 24 & 23.9 \\
7.7 & $0.7^{*}$ & 25 & 23.2 \\
7.8 & 0.9 & 31 & $15.0^{*}$
\end{tabular}

Note: For the second fit the value of $u_{33}$ was fixed to $0.7 \AA^{2}$, for the third fit the value of $d_{\mathrm{sp}}$ was fixed to $15 \AA$. Both fixings are denoted by an asterisk. 


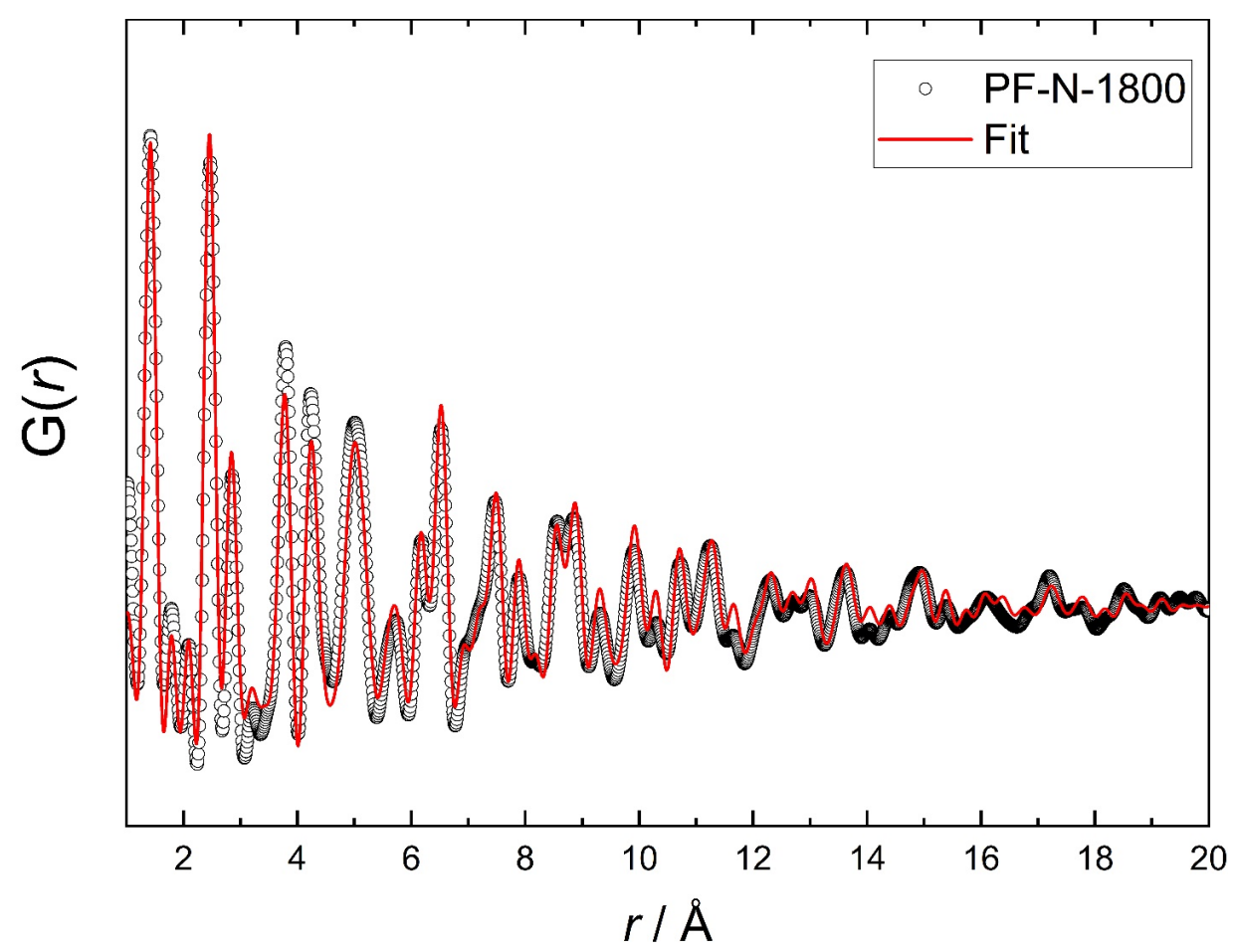

Figure S7: Fit of the PDF data of the PF-N-1800 sample up to $20 \AA$ without restraints. A $s p$ diameter of $23.9 \AA$ is obtained.

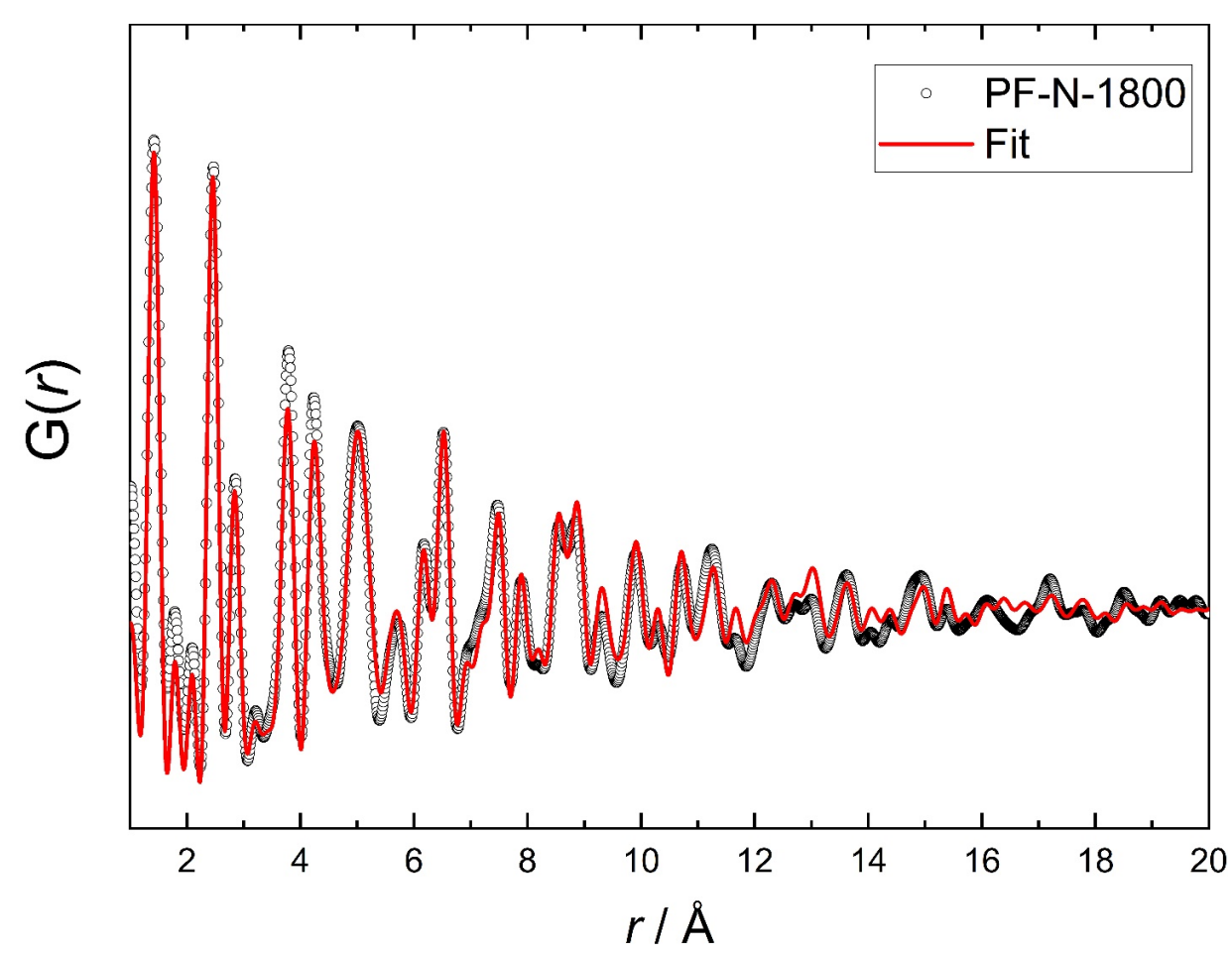

Figure S8: Fit of the PDF data of the PF-N-1800 up to $20 \AA$ sample with $u_{33}$ fixed to $0.7 \AA^{2}$. A $s p$ diameter of $23.2 \AA$ is obtained, similarly to the sp diameter obtained by fitting without constraints. 


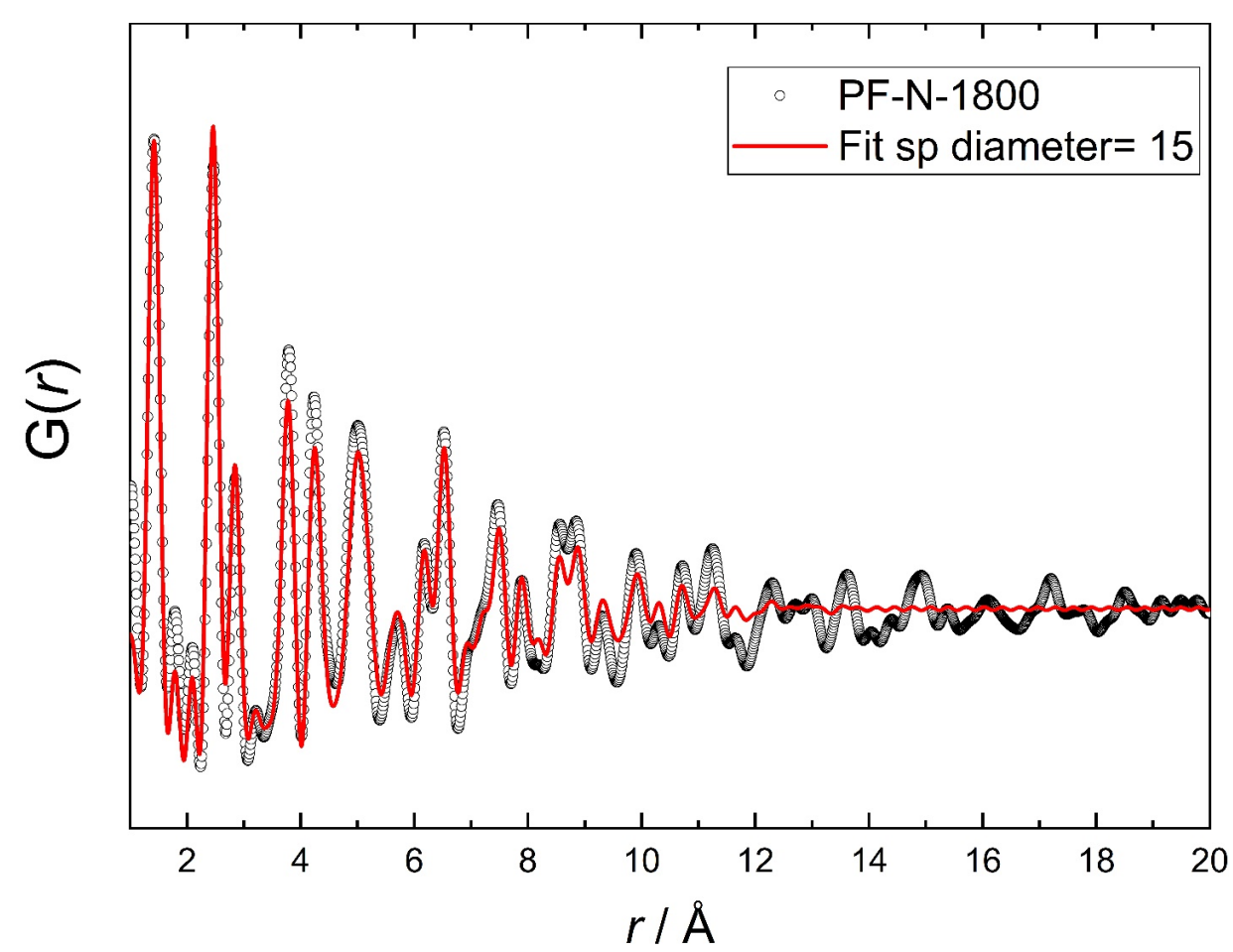

Figure S9: Fit of the PDF data of the PF-N-1800 sample up to $20 \AA$ with $d_{\text {sp }}$ fixed to $15.0 \AA$. The lower $s p$ diameter causes a onset of the dampening at a significant lower $r$-value.

Table S6: Overview of the parameters obtained by fitting the PDF data up to $20 \AA$ and $80 \AA$ (the latter given in brackets) as described in ${ }^{1}$ along with the corresponding $L_{\mathrm{a}}$ values. For the AC-2400 and LSPP-1800 sample, the bold marked values are assumed to be more realistic as for these samples the PDF signal dampens at $r>20 \AA$.

\begin{tabular}{|c|c|c|c|c|c|}
\hline sample & $c / \AA$ & $u_{33} / \AA^{2}$ & $R_{\mathrm{w}} / \%$ & $d_{\mathrm{sp}} / \AA$ & $L_{\mathrm{a}}(\mathrm{WAXS}) / \AA$ \\
\hline AC-2400 & $\begin{array}{c}6.8 \\
(6.8)\end{array}$ & $\begin{array}{c}0.10 \\
(\mathbf{0 . 1 5})\end{array}$ & $\begin{array}{c}0.34 \\
(\mathbf{0 . 4 2})\end{array}$ & $\begin{array}{c}35 \\
(50)\end{array}$ & $\mathrm{n} / \mathrm{a}$ \\
\hline PF-N-1800 & $\begin{array}{c}8.0 \\
(7.9)\end{array}$ & $\begin{array}{c}1.45 \\
(1.46)\end{array}$ & $\begin{array}{c}0.24 \\
(0.25)\end{array}$ & $\begin{array}{c}24 \\
(24)\end{array}$ & 42 \\
\hline LSSP-1800 & $\begin{array}{c}6.9 \\
(6.9) \\
\end{array}$ & $\begin{array}{c}0.17 \\
(\mathbf{0 . 2 1})\end{array}$ & $\begin{array}{c}0.51 \\
(\mathbf{0 . 6 1}) \\
\end{array}$ & $\begin{array}{c}32 \\
(\mathbf{4 1}) \\
\end{array}$ & 163 \\
\hline LSPP-1200 & $\begin{array}{c}7.1 \\
(7.1)\end{array}$ & $\begin{array}{c}0.22 \\
(0.22)\end{array}$ & $\begin{array}{c}0.29 \\
(0.30)\end{array}$ & $\begin{array}{c}19 \\
(19)\end{array}$ & 39 \\
\hline LSPP-800 & $\begin{array}{c}7.9 \\
(8.0) \\
\end{array}$ & $\begin{array}{c}1.36 \\
(1.40)\end{array}$ & $\begin{array}{c}0.40 \\
(0.41)\end{array}$ & $\begin{array}{c}13 \\
(13)\end{array}$ & 17 \\
\hline CB-800 & $\begin{array}{c}8.0 \\
(7.9)\end{array}$ & $\begin{array}{c}2.85 \\
(1.94)\end{array}$ & $\begin{array}{c}0.52 \\
(0.54)\end{array}$ & $\begin{array}{c}12 \\
(11)\end{array}$ & 26 \\
\hline PF-N-800 & $\begin{array}{c}8.5 \\
(8.4)\end{array}$ & $\begin{array}{c}1.69 \\
(1.29)\end{array}$ & $\begin{array}{c}0.35 \\
(0.36)\end{array}$ & $\begin{array}{c}13 \\
(13)\end{array}$ & 20 \\
\hline
\end{tabular}

\section{References}

1. Poulain, A.; Dupont, C.; Martinez, P.; Guizani, C.; Drnec, J. Wide-angle X-ray scattering combined with pair distribution function analysis of pyrolyzed wood. Journal of Applied Crystallography 2019, 52, 60-71. 